$$
\int_{0}^{1}\left\{\frac{l_{1}(x)}{\kappa}+r_{1}(x)\right\} u_{1}^{2}(x) d x=\frac{1}{\kappa} .
$$

By taking $\kappa$ large enough it is possible to choose $u_{1}(x)$ (in an infinite number of ways) such that equation (25) is satisfied and $\left|u_{1}(x)\right|<\epsilon,\left|u_{1}^{\prime}(x)\right|<\epsilon$, where $\epsilon$ is an arbitrarily small positive number. For this value of $u_{1}(x)$ (and $\left.u_{2}(x) \equiv 0\right), D\left(u_{1}, u_{2}\right)<C \epsilon^{2}$, where $C$ is a constant such that $C>p_{1}(x)-q_{1}(x)$. The value of $\kappa$ is then so chosen that $m(\kappa)$ is less then $C^{\prime} \epsilon^{2}$. In a similar manner it may be shown that $m(-\infty)=0$.

Brown University, Providence, September, 1910.

\title{
NOTE ON IDENTITIES CONNECTING CERTAIN INTEGRALS.
}

BY DR. LOUIS INGOLD.

(Read before the American Mathematical Society, Septєmber 7, 1910.)

Because of the general nature of the symbols and symbolic products used in the symbolic invariant theory, it is possible to apply the formulas of this theory in various special fields. In the present note the theory is employed to obtain relations connecting integrals of functions constructed out of a linearly independent set.

1. We shall be interested in functions of $n$ parameters $u_{1}$, $u_{2}, \cdots, u_{n}$ and functions of one or more real variables $x, x_{1}$, $x_{2}, \cdots, y, y_{1}, y_{2}, \cdots$, etc., restricted to a definite interval, say, $0 \leq x \leq 1$. In order to distinguish readily between those quantities which are constant or functions of the parameters $u_{1}, \cdots, u_{n}$ only, and those which are functions of one or more of the variables $x, x_{1}, x_{2}, \cdots$, etc., the latter will always be denoted by black faced letters; the others by letters in ordinary type; thus a denotes a function of a variable $x$ defined for values of $x$ on the interval $0 \ldots 1$, while $a$ denotes a function of the $u_{i}$ only or a constant. The partial derivatives $\partial \mathrm{a} / \partial u_{i}$, $\partial a / \partial u_{i}$, etc., will be denoted by $\mathbf{a}_{(i)}, a_{(i)}$, etc.

2. Consider now the total differential of any function $\mathrm{f}\left(x ; u_{1}\right.$, $u_{2}, \cdots, u_{n}$ ) with respect to the $u_{i}$

$$
d \mathbf{f}=\mathbf{f}_{(1)} d u_{1}+\mathbf{f}_{(2)} d u_{2}+\cdots+\mathbf{f}_{(n)} d u_{n} .
$$


Let this expression be squared and integrated with respect to $x$ from 0 to 1 . We obtain in this way the quadratic differential form in the variables $u_{1}, \cdots, u_{n}$

$$
\begin{aligned}
\int_{0}^{1}(d \mathbf{f})^{2} d x & =\int_{0}^{1}\left(\mathbf{f}_{(1)} d u_{1}+\mathbf{f}_{(2)} d u_{2}+\cdots+\mathbf{f}_{(n)} d u_{n}\right)^{2} d x \\
& =\sum_{i, j=1}^{n}\left(\int_{0}^{1} \mathbf{f}_{(i)} \mathbf{f}_{(j)} d x\right) d u_{i} d u_{j}=\sum_{i, j=1}^{n} E_{i j} d u_{i} d u_{j},
\end{aligned}
$$

where the expressions

$$
E_{i j}=\int_{0}^{1} \mathbf{f}_{(i)} \mathbf{f}_{(j)} d x
$$

are functions of $u_{1}, u_{2}, \cdots, u_{n}$.

3. If now we introduce a symbolic multiplication equivalent to ordinary multiplication followed by integration with respect to $x$ from 0 to 1 , we may write

$$
\begin{aligned}
\overline{d \mathbf{f}^{2}} & =\left(\overline{\mathbf{f}}_{(1)} d u_{1}+\mathbf{f}_{(2)} d u_{2}+\cdots+\mathbf{f}_{(n)} d u_{n}\right)^{2} \\
& =\sum_{i, j=1}^{n} \overline{\mathbf{f}_{(i)} \mathbf{f}_{(j)}} d u_{i} d u_{j}=\sum_{i, j=1}^{n} E_{i j} d u_{i} d u_{j},
\end{aligned}
$$

where the bars indicate that the products are symbolic, i. e., integrated products. We have thus expressed the quadratic differential form

$$
E \equiv \sum_{i, j=1}^{n} E_{i j} d u_{i} d u_{j}
$$

as the symbolic square of a linear form.*

4. It should be noticed that we have also

$$
\int_{0}^{1}[d \mathrm{f}(y)]^{2} d y=\int_{0}^{1}[d \mathrm{f}(x)]^{2} d x=E
$$

and again, if $\phi\left(x ; u_{1}, u_{2}, \cdots, u_{n}\right)$ is any function satisfying the conditions

$$
\int_{0}^{1} \phi_{(i)} \phi_{(j)} d x=E_{i j}
$$

then

$$
\int_{0}^{1}\left[(d \phi(x)]^{2} d x=E .\right.
$$

* Cf. Maschke, "A symbolic treatment of the theory of invariants of quadratic differential quantics of $n$ variables," Transactions, vol. 4 (1904), pp. 445-469. 
Such equivalent expressions for $E$ may be employed in a manner analogous to the use of equivalent symbols in the symbolic theory.* In this paper the method indicated in equations (5) will be used for equivalent representation of $E$.

5 . On account of the linear distributive character of integration, the ordinary function $\mathbf{f}\left(x ; u_{1}, u_{2}, \ldots, u_{n}\right)$ satisfying conditions (3) may be taken as a symbolic function or symbol of the differential form $E$, and all the operations of the symbolic theory can be carried out if we agree that in products no more than two factors shall be expressed in terms of the same variable $x$; that products of two factors written in terms of the same variable $x$ shall be regarded as integrated products; and that a product of any number of factors expressed in terms of different variables $x, x_{1}, x_{2}, \cdots, y, y_{1}, y_{2}, \cdots$ shall be regarded as ordinary products.

We have then this result:

All of the identities of the symbolic differential invariant theory $\dagger$ may be interpreted as identities involving ordinary functions and their integrals.

6 . The identities of the symbolic theory are given mainly in terms of determinants of the $n$th order. We denote the determinant

$$
\left|\int_{0}^{1} f_{(i)} f_{(j)} d x\right| \text { by } \frac{1}{\beta^{2}} .
$$

The product of $\beta$ and the Jacobian of $n$ functions $a_{1}, a_{2}, \ldots, a_{n}$, is denoted by

$$
\left(a_{1}, a_{2}, \cdots, a_{n}\right)
$$

Using these notations we may write, for example, the symbolic expression

$\left(f_{1}, f_{2}, \cdots, f_{k}, a_{1}, a_{2}, \cdots, a_{n-k}\right)\left(f_{1}, f_{2}, \ldots, f_{k}, b_{1}, b_{2}, \ldots, b_{n-k_{k}}\right)$ (where $f_{1}, f_{2}, \cdots, f_{k}$ are equivalent symbolic functions of the differential quantic $E$ ) in the form

$$
\begin{aligned}
& \int_{0}^{1}(k)\left[\mathrm{f}\left(x_{1}\right), \mathrm{f}\left(x_{2}\right), \cdots, \mathrm{f}\left(x_{l_{k}}\right), a_{1}, \ldots, a_{n-k}\right] \\
& \times\left[\mathrm{f}\left(x_{1}\right), \cdots, \mathrm{f}\left(x_{k}\right), b_{1}, \cdots, b_{n-k}\right] d x_{1}, \cdots, d x_{k},
\end{aligned}
$$

* Maschke, loc. cit., p. 448.

† Clearly, the result holds equally well for the algebraic invariant theory. 
7. Out of the large number of identities which could now be written down, we select the following for illustration.

$$
\begin{aligned}
& \int_{0}^{1}{ }^{(n-k)}\left[\mathrm{f}\left(x_{1}\right), \cdots, \mathrm{f}\left(x_{n-k}\right), a_{1}, \cdots, a_{k}\right] \\
& \times\left[\mathrm{f}\left(x_{1}\right), \ldots, \mathrm{f}\left(x_{n-k}\right), b_{1}, \cdots, b_{l k}\right] d x_{1}, \cdots, d x_{n-k} \\
& =\frac{(n-k) !}{(n-1) !} \mid \int_{0}^{1}(n-1)\left[\mathrm{f}\left(x_{1}\right), \cdots, \mathrm{f}\left(x_{n-1}\right), a_{i}\right] \\
& \times\left[\mathrm{f}\left(x_{1}\right), \cdots, \mathrm{f}\left(x_{n-1}\right), b_{j}\right] d x_{1}, \cdots, d x_{n-1} \mid, *
\end{aligned}
$$

where the determinant on the right is of the $k$ th order, $i$ and $j$ running from 1 to $k$.

$$
\begin{aligned}
\int_{0}^{{ }^{1}(k)}\left[\mathrm{f}\left(x_{1}\right),\right. & \left.\cdots, \mathrm{f}\left(x_{k}\right), \mathrm{f}\left(x_{k+1}\right), \cdots, \mathrm{f}\left(x_{n}\right)\right] \\
& \times\left[\mathrm{f}\left(x_{1}\right), \cdots, \mathrm{f}\left(x_{k}\right), a_{1}, \cdots, a_{n-k}\right] \\
& \times\left[\mathrm{f}\left(x_{k+1}\right), \cdots, \mathrm{f}\left(x_{n}\right), b_{1}, \cdots, b_{k}\right] d x_{1}, \cdots, d x_{n} \\
= & k !(n-k) !\left(a_{1}, \cdots, a_{n-k}, b_{1}, \cdots, b_{k}\right) \cdot \uparrow
\end{aligned}
$$

$$
\begin{aligned}
& \int_{0}^{1}{ }^{(n-k-r)}\left[\mathrm{f}\left(x_{1}\right), \cdots, \mathbf{f}\left(x_{k}\right), \mathbf{f}\left(x_{k+1}\right), \cdots, \mathbf{f}\left(x_{n-r}\right), b_{1}, \cdots, b_{r}\right] \\
& \quad \times\left[\mathrm{f}\left(x_{k+1}\right), \cdots, \mathbf{f}\left(x_{n-r}\right), a_{1}, \cdots, a_{k+r}\right] d x_{k+1}, \cdots, d x_{n-r}
\end{aligned}
$$$$
\text { (9) }=\frac{(n-k-r) !}{(n-k) !(n-r) !} \sum_{i} \int_{0}^{{ }^{1}(n-r)}\left[\mathrm{f}\left(x_{1}\right), \ldots, \mathbf{f}\left(x_{n-r}\right), a_{i}, \cdots, a_{i_{r}}\right]
$$

$$
\begin{aligned}
& \times\left[\mathrm{f}\left(x_{1}\right), \cdots, \mathbf{f}\left(x_{n-r}\right), b_{1}, \cdots, b_{r}\right] d x_{k+1}, \cdots, d x_{n-r} \\
& \times \int_{0}^{(n-r)}\left[\mathrm{f}\left(y_{1}\right), \cdots, \mathbf{f}\left(y_{n-k}\right), a_{i_{1+r}}, \cdots, a_{i_{k+r}}\right] \\
& \times\left[\mathbf{f}\left(y_{1}\right), \cdots, \mathbf{f}\left(y_{n-k_{k}}\right) \mathbf{f}\left(x_{1}\right), \cdots, \mathbf{f}\left(x_{l_{k}}\right)\right] d y_{1}, \cdots, d y_{n-k}, \downarrow
\end{aligned}
$$

* Maschke, "Differential parameters of the first order," Transactions, vol. 7 (1906), p. 74, formula (8).

+ Cf. Maschke, loc. cit., p. 453 , formula (34) and p. 455 , formula (61). Only the cases $k=1$ and $k=2$ are given by Maschke but this extension is easily proved.

† This formula is given in my paper "Vector interpretation of symbolic differential parameters," Transactions, vol. 11, p. 470. 
the summation extending over all distinct combinations $i_{1} \ldots, i_{r}$ of $r$ of the subscripts $1,2, \cdots, k+r$ and the order $i_{1}, i_{2}, \cdots$, $i_{k+r}$ being so chosen that it may be obtained from $1,2, \cdots, k+r$ by an even number of transpositions.

8. We now consider a special case in which $f$ is linear in the $u_{i}$ and a set of $n$ normed and mutually orthogonal functions $\mathrm{f}_{(1)}, \mathrm{f}_{(2)}, \cdots, \mathbf{f}_{(n)}$, of the variable $x$

$$
\mathbf{f}=u_{1} \mathbf{f}_{(1)}+u_{2} \mathbf{f}_{(2)}+\cdots+u_{n} \mathbf{f}_{(n)} .
$$

We shall also suppose the functions $a_{i}, b_{i}$, etc., of the $u$ 's to be linear, and write

$$
a_{i}=\sum_{j=1}^{n} \alpha_{i j} u_{j}, \quad b_{i}=\sum_{j=1}^{n} \beta_{i j} u_{j}, \quad \text { etc. }
$$

where the coefficients $\alpha_{i j}, \beta_{i j}$, etc., are constant.

With every linear function $a_{i}$ of the $u$ 's we shall associate a function $a_{i}(x)$, defined by

$$
a_{i}(x)=\sum_{j=1}^{n} \alpha_{i j} f_{(i j)}
$$

We then introduce the notations

$$
\begin{aligned}
\mathbf{A}_{\left(x_{1}, \ldots, x_{k}\right)} & =\frac{1}{k !}\left|\begin{array}{l}
\mathrm{a}_{1}\left(x_{1}\right), \cdots, \mathrm{a}_{1}\left(x_{l_{k}}\right) \\
\mathrm{a}_{2}\left(x_{1}\right), \cdots, \mathrm{a}_{2}\left(x_{k_{k}}\right) \\
\mathrm{a}_{l_{k}}\left(x_{1}\right), \cdots, \mathrm{a}_{l k}\left(x_{l_{k}}\right)
\end{array}\right| \\
\mathbf{F}\left(x_{1}, \ldots, x_{n}\right) & =\left[\mathrm{f}\left(x_{1}\right), \mathrm{f}\left(x_{2}\right), \ldots, \mathrm{f}\left(x_{n}\right)\right] .
\end{aligned}
$$

We define the complement of $\mathbf{A}$ (written $\overline{\mathbf{A}}$ ) by the formula

$$
\begin{aligned}
& (\mathbf{A})_{\left(x_{1}, \ldots, x_{n-k}\right)} \\
& \quad=\int_{0}^{{ }^{(}\left(k_{)}\right)} \mathbf{F}\left(y_{1}, \ldots, y_{k}, x_{1}, \cdots, x_{n-k}\right) \mathbf{A}_{\left(y_{1}, \ldots, y_{k}\right)} d y_{1}, \cdots, d y_{k} .
\end{aligned}
$$

The following formula may then be easily proved :

$$
\begin{aligned}
& k !(n-k) ! \mathbf{A}_{\left(x_{1}, \ldots, x_{k}\right)} \\
&= \int_{0}^{1}{ }^{(n-k)}\left[\mathrm{f}\left(y_{1}\right), \ldots, \mathrm{f}\left(y_{n-k}\right), \mathbf{f}\left(x_{1}\right), \ldots, \mathrm{f}\left(x_{k}\right)\right] \\
& \quad \times\left[\mathrm{f}\left(y_{1}\right), \ldots \mathrm{f}\left(y_{n-k}\right), a_{1}, \ldots, a_{k}\right] d y_{1}, \cdots, d y_{n-k}, \\
& \mathbf{A}=\frac{1}{k ![(n-k) !]^{3}} \int_{0}^{(k)} \mathbf{F}\left(y_{1}, \cdots, y_{k} x_{1}, \cdots, x_{n-k}\right)
\end{aligned}
$$




$$
\begin{aligned}
& \times \int_{0}^{1}{ }_{(n-k)} \mathbf{F}\left(z_{1}, \cdots, z_{n-k} y_{1}, \cdots, y_{k_{0}}\right) \\
& \times\left[\mathrm{f}\left(z_{1}\right), \ldots, \mathrm{f}\left(z_{n-k}\right), a_{1}, \cdots, a_{k}\right] d z_{1}, \cdots, d z_{n-k} d y_{1}, \cdots, d y_{k} \\
& = \pm \frac{1}{(n-k) !}\left[\mathrm{f}\left(x_{1}\right), \cdots, \mathrm{f}\left(x_{n-k}\right), a_{1}, \cdots, a_{k}\right] \text {. } \\
& \overline{\overline{\mathbf{A}}}= \pm \mathbf{A} \text {. } \\
& \int_{0}^{1}{ }^{1} \mathbf{A}_{\left(x_{1}, \ldots, x_{k}\right)} \mathbf{B}_{\left(x_{1}, \ldots, x_{k}\right)} d x_{1}, \cdots, d x_{k} \\
& \times \int_{0}^{1}(n-k)(\overline{\mathbf{A}})_{\left(x_{1}, \ldots, x_{n-k)}\right.}(\overline{\mathbf{B}})_{\left(x_{1}, \ldots, x_{n-k}\right)} d x_{1}, \cdots, d x_{n-k} \text {. }
\end{aligned}
$$

Substituting in formulas (7), (8), and (9), we have

$\left(7^{\prime}\right) \quad \int_{0}^{1}{ }^{(k)} \mathbf{A}_{\left(x_{1}, \ldots, x_{k}\right)} \mathbf{B}_{\left(x_{1}, \ldots, x_{k}\right)} d x_{1}, \ldots, d x_{k}=\left|\int_{0}^{1} \mathbf{a}_{i} \mathbf{b}_{j} d x\right|,{ }^{*}$

$\left(8^{\prime}\right) \int_{0}^{1}{ }^{1}(n-k) \mathbf{A}_{\left(x_{1}, \ldots, x_{n-k}\right)}(\overline{\mathbf{B}})_{\left(x_{1}, \ldots, x_{n-k}\right)} d x_{1}, \ldots, d x_{n-k}$ $=(-1)^{k(n-k)} \int_{0}^{1}{ }^{(k)}(\overline{\mathbf{A}})_{\left(x_{1}, \ldots, x_{k}\right)} \mathbf{B}_{\left(x_{1}, \ldots, x_{k}\right)} d x_{1}, \ldots, d x_{k}$,

$$
\begin{aligned}
\left(9^{\prime}\right) & \int_{0}^{1}(j)(\overline{\mathbf{A}})_{\left(y_{1}, \ldots, y_{j}\right)}(\overline{\mathbf{B}})_{\left(x_{1}, \ldots, x_{k} y_{1}, \ldots, y_{j}\right)} d y_{1}, \ldots, d y_{j} \\
& =\frac{k ! j !}{(k+j) !} \sum_{i} \mathbf{A}_{i\left(x_{1}, \ldots, x_{k}\right)} \int_{0}^{1}\left({ }^{1}\right)\left(\overline{\mathbf{A}}_{i}\right)_{\left(y_{1}, \ldots, y_{j}\right)}(\overline{\mathbf{B}})_{\left(y_{1}, \ldots, y_{j}\right)} d y_{1}, \ldots, d y_{j}
\end{aligned}
$$

where $k+j=n-r$ and where $i$ has the same meaning as in formula (9).

UNIVERSITY OF MissourI, July, 1910 .

* This identity is proved directly by Professor R. G. D. Richardson and Dr. W. A. Hurwitz in the paper "Note on determinants whose terms are certain integrals," Bulletis, vol. 16, p. 16 . 